\title{
Physical properties of a planar magnetron glow discharge
}

\author{
Ketan Trivedi, Stephen Brewer, ${ }^{*}$ Lori McCaig, Norman Sesi and Richard Sacks $\dagger$ \\ Department of Chemistry, University of Michigan, Ann Arbor, MI 48109 U.S.A.
}

(Received 30 May 1990; accepted 20 August 1990)

\begin{abstract}
Continued work with a planar magnetron glow discharge plasma device which uses permanent magnets located behind a planar cathode to trap plasma electrons in circular orbits parallel to the cathode surfacc is described. This results in a significant reduction in plasma voltage, and stable operation is achieved over an extended range of pressures and cathode current densities. The plasma has the form of a ring extending from the cathode surface to several millimeters from the surface. The plasma geometry is determined by the locus of greatest magnetic field strength. Cathode current density values greater than $100 \mathrm{~mA} / \mathrm{cm}^{2}$ are easily achieved for pressures in the range 0.0007 Torr $(0.093 \mathrm{~Pa})$ to over 2.5 Torr (330 $\mathrm{Pa}$ ). Over this range, the plasma voltage is under $500 \mathrm{~V}$. Spatially-resolved emission spectral data are presented showing the effects of plasma current and pressure on line intensities from plasma gas species as well as from cathodically-sputtered species. Data are presented for a pure $\mathrm{Cu}$ cathode and for a $\mathrm{Zn}$-based alloy used as a standard reference material.
\end{abstract}

\section{INTRODUCTION}

THE USE of glow discharge plasma devices is growing rapidly in several important areas including chemical analysis and chemical processing. For chemical analysis applications, the glow discharge plasma has been used as an atom source for atomic absorption [1-3] and atomic fluorescence [4,5] spectrometry, as an excitation source for atomic emission spectroscopy [6-10], and as an ion source for mass spectrometry [11-15]. The usefulness of the glow discharge plasma depends on the rates and efficiencies of the processes responsible for the formation of sample atoms, excited states and ions.

A variety of techniques are being investigated for obtaining greater efficiencies and greater control of these processes. Noteworthy examples include the use of auxiliary gas jets aimed at the cathode surface [16-18], the use of pulsed and radio-frequency plasma formation techniques $[19,20]$ and the use of magnetic fields to alter plasma structure [21-23] and the rate of charge transport in the plasma $[24,25]$.

In the presence of an externally-applied magnetic field, charged particles in the plasma precess around magnetic field lines, and the rate of charge transport normal to the magnetic field direction is reduced relative to its isotropic value $[24,26]$. For the field strengths usually used, only plasma electrons are significantly affected by the field. The presence of curved magnetic field lines, a magnetic field gradient, or a macroscopic electric field normal to the magnetic field results in electrons undergoing additional drift motions [26].

In a magnetron glow discharge, these drift motions take the form of closed loops parallel to the cathode surface [27-31]. This results in an increase in the effective electron path length and a corresponding increase in the efficiency of plasma gas ion formation by impact with high-kinetic-energy electrons. The plasma is confined to the regions of greatest magnetic field strength, and only in these regions is significant cathodic sputtering observed. Greater ionization efficiency of the plasma gas results in significantly lower plasma voltage [29] and improved performance at lower pressures [30,31].

Magnetron glow discharge devices using planar cathodes and designed for chemical analysis were recently described $[32,33]$. Detailed descriptions of magnetic field

\footnotetext{
* Chemistry Dept, Eastern Michigan University, Ypsilanti, MI 48197, U.S.A.

+ Author to whom correspondence should be addressed.
} 
distributions and polarities also were given. The latter study describes the use of a concentric pair of permanent magnets located behind the cathode in order to trap plasma electrons in ring-shaped orbits near the cathode surface. This device operates over a pressure range from less than 0.0007 Torr $(0.093 \mathrm{~Pa})$ to over 2.5 Torr $(330 \mathrm{~Pa})$, and over this entire range, average cathode current densities in the sputter track greater than $100 \mathrm{~mA} / \mathrm{cm}^{2}$ can be obtained with plasma voltage less than $500 \mathrm{~V}$. This configuration, described in detail in Ref. [33], was used in the present study.

In this report, spatially-resolved emission spectroscopy is used to characterize the plasma structure of the planar magnetron and to determine the sensitivity of this structure to the plasma current and the operating pressure. Data are presented for cathodes of pure $\mathrm{Cu}$ and for $\mathrm{Zn}$-based alloy disks mounted in the $\mathrm{Cu}$ cathode.

\section{EXPERIMENTAL}

\subsection{Magnetron design}

Figure 1 shows the important conceptual features of the planar magnetron system used for this study. Part (a) shows a side section view of the electrode assembly, and part (b) shows the orientation of the device with respect to the spectrometer entrance slit and optical system. Components and construction details are found in Table 1. A more detailed description of the system is found in Ref. [33].

The $\mathrm{Cu}$ cathode plate $\mathrm{C}$ is about $9 \mathrm{~cm}$ in diameter and is mounted on the end of an insulating, cylindrical, water-cooled chamber (not shown in the figure). Located in the chamber and flat against the back of the cathode is a concentric pair of permanent magnets. The inner magnet $M_{1}$ is a $\mathrm{Co} / \mathrm{Sm}$ rare-earth disk which produces a high flux density at the cathode surface. The outer magnet $M_{2}$ is a ceramic ring. This magnet reduces the fringing fields from the Co/Sm disk, and the combination of the two magnets allows for lower pressure operation than either magnet alone $[32,33]$. The cold-drawn steel pole piece $D$ results in higher flux density at the cathode surface. The anode plate $A$ is a stainless steel disk. The anode-cathode separation is about $10 \mathrm{~mm}$.

The plasma $\mathbf{P}$ has the form of a ring with a mean diameter of about $25 \mathrm{~mm}$ and extending several $\mathrm{mm}$ from the cathode surface. Sputtering is observed only in the ring-shaped track $\mathrm{T}$

(a)

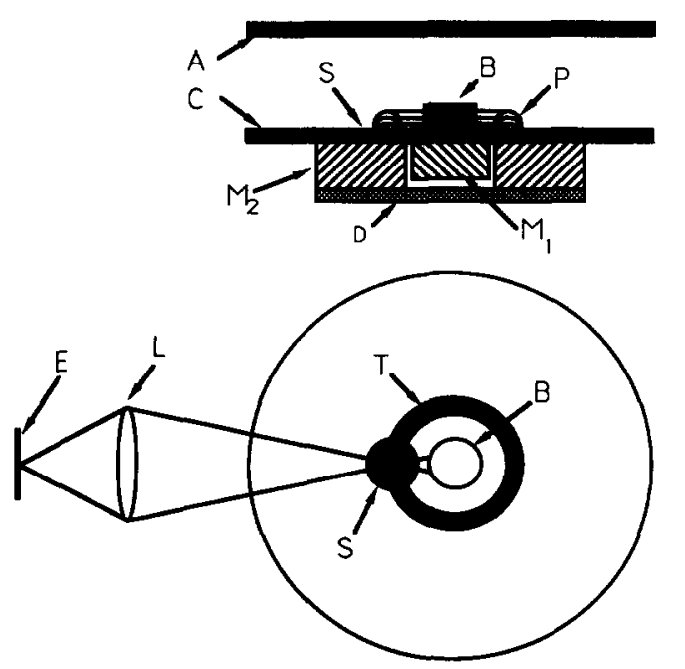

Fig. 1. (a) Side-section view of the planar magnetron device. A, Anode disk; C, cathode disk; $P$, ring-shaped plasma: $M_{1}$, disk-shaped rare-earth magnet; $M_{2}$, ring-shaped ceramic magnet; $\mathrm{D}$, pole piece; S, sample disk; B, mask to block radiation from the portion of the plasma ring across from the sample disk. (b) View of the device normal to the cathode surface showing the sputter track $T$ intersecting the sample disk. The cathode surface is parallel to the spectrometer entrance slit $\mathrm{E}$, and a segment of the plasma ring is imaged onto the slit by lens $\mathrm{L}$ 
Table 1. Construction details of the planar magnetron device

\begin{tabular}{|c|c|}
\hline Cathode & $\begin{array}{l}\text { Copper disk: } 9-\mathrm{cm} \text { dia., } 1.6 \mathrm{~mm} \text { thick with } 9.5-\mathrm{mm} \text { dia., } \\
6.4-\mathrm{mm} \text { long center post and } 9.5-\mathrm{mm} \text { dia. sample hole }\end{array}$ \\
\hline Backing chamber & $\begin{array}{l}\text { Water-cooled copper cylinder: } 9-\mathrm{cm} \text { dia., } 2.9-\mathrm{cm} \text { long } \\
\text { with } 1.6-\mathrm{mm} \text { thick backing plate }\end{array}$ \\
\hline Inner magnet & $\begin{array}{l}\text { Cobalt-samarium rare-earth disk: } 19-\mathrm{mm} \text { dia., } 6.4-\mathrm{mm} \\
\text { thick; Magnet Sales and Manufacturing Co., Culver City, } \\
\mathrm{CA}\end{array}$ \\
\hline Outer magnet & $\begin{array}{l}\text { Ferrite-based ceramic ring: } 60.3-\mathrm{mm} \text { o.d., } 21.6-\mathrm{mm} \text { i.d., } \\
13-\mathrm{mm} \text { thick; Edmund Scientific, Barrington, NJ }\end{array}$ \\
\hline Pole & Cold-drawn steel disk: $6.0-\mathrm{cm}$ dia, $16-\mathrm{mm}$ thick \\
\hline Anode & $\begin{array}{l}\text { Stainless steel disk: } 9-\mathrm{cm} \text { dia., } 1.6-\mathrm{mm} \text { thick; location, } 10 \\
\mathrm{~mm} \text { from cathode }\end{array}$ \\
\hline Chamber & Welded stainless steel cylinder, $16.2-\mathrm{cm}$ i.d., $25.4-\mathrm{cm}$ long \\
\hline
\end{tabular}

shown in part (b) of Fig. 1. The surface area sputtered is about $4 \mathrm{~cm}^{2}$. A $9.5-\mathrm{mm}$ hole in the cathode plate is used to accommodate a sample pellet $S$. The hole intersects the sputter track. The pellet is pressed into the hole so that the pellet surface is coplanar with the cathode surface.

In part (b) of Fig. 1, the cathode surface is shown in its normal operating configuration with the surface parallel to the spectrometer entrance slit $E$. Lens $L$ is used to image the plasma onto the entrance slit. A post B, located at the center of the cathode, blocks radiation from the portion of the plasma ring across a diameter from the sample pellet. This improves spatial resolution and reduces background radiation. The presence of the post results in no observable change in the plasma properties or structure.

The electrode assembly is located in a stainless steel chamber equipped with quartz observation windows, plasma gas inlet, vacuum-line port and pressure gauges. The electrode assembly is attached to a mechanical, high-vacuum feedthrough which passes through the cylindrical chamber wall. This permits the electrode assembly to be translated normal to the electrode axis (normal to the plane of Fig. 1b) during plasma operation. This feature is used to obtain spectra with spatial resolution normal to the cathode surface.

The chamber is pumped by a 4-in. oil diffusion pump backed by a two-stage mechanical pump. An $\mathrm{LN}_{2}$ baffle located between the chamber and the diffusion pump is used to reduce back-streaming of pump oil. A thermocouple gauge is used to monitor chamber pressure for values between 0.02 Torr and 2.5 Torr. Air-to-Ar calibration conversions were provided by the manufacturer. A cold-cathode ionization gauge is used for lower pressures. The latter gauge was calibrated with a high-resolution McLeod gauge.

\subsection{Optical and electrical monitoring}

All spectral data were obtained with a 1.0-m Czerny-Turner spectrometer (Jarrell-Ash Model 78-462) equipped with a 600 line $/ \mathrm{mm}$ grating blazed for $500 \mathrm{~nm}$ in the first order. Entrance and exit slit widths ranged from $40-100 \mu \mathrm{m}$. Radiation intensity measurements were obtained with a 1P28 photomultiplier tube. Bias voltage and load resistance were adjusted as needed. A 100$\mathrm{mm}$ focal length, 38-mm dia. quartz lens was used to image the plasma onto the entrance slit with a lateral magnification of 0.15 . With a $50 \mu \mathrm{m}$ entrance slit width, the spatial resolution is about $0.33 \mathrm{~mm}$ along an axis normal to the cathode surface and passing through the center of the sputter track. The $f / 24$ aperture ratio used here results in significantly reduced optical aberrations. Magnetic field measurement techniques are described in Ref. [32].

\subsection{Procedures and materials}

The Ar plasma gas was passed through filters for water vapor, oxygen and hydrocarbons and finally through an $\mathrm{LN}_{2}$ trap. Sample pellets $(9.5-\mathrm{mm}$ dia., $1.6-\mathrm{mm}$ thick) were machined from regions of NIST zinc-base alloys (Standard Reference Materials 625-630) certified for minor and trace element composition and fitted into the hole machined in the $\mathrm{Cu}$ cathode shown in Fig. 1. A strip-chart recorder was used to record intensity data. Background intensity values were recorded at wavelengths near those of peak line intensities used for quantitative work. 


\section{Results ANd Discussion}

\subsection{General plasma characteristics}

The plasma appears as a thin bright ring anchored to the cathode surface and a more diffuse region extending at least several millimeters from the cathode. If a dark space is present at the cathode surface, it is very thin, probably less than $1 \mathrm{~mm}$, over the entire pressure range studied. The plasma was stable at all pressures in the range 0.0007 Torr to over 2.5 Torr. At pressures greater than about 0.15 Torr, a secondary plasma ring was observed outside of the primary ring, but no sputtering was observed where the secondary ring contacted the cathode surface. For pressures greater than about 0.02 Torr, the anode disk could be removed, and the grounded chamber wall served as the anode. This produced no discernible change in the structure of the bright plasma ring. All measurements reported here were made with the anode disk present in order to ensure a consistent electrode configuration over the entire useful pressure range.

An important potential advantage of the planar magnetron device for chemical analysis applications is the lower operating pressure which may reduce interferences from background gas species. Figure 2 shows the effects of Ar pressure and pumping speed on the intensities of some band features in the wavelength range 387-392 nm. Spectrum (a) was obtained at a chamber pressure of 0.17 Torr. The chamber was pumped by a two-stage mechanical pump. With this pump, the ultimate chamber pressure with no Ar flow into the chamber is about 0.02 Torr. Spectrum (b) was obtained at a pressure of 0.08 Torr. Again, the mechanical pump was used. Spectrum (c) also was obtained at 0.08 Torr, but the chamber was pumped by a 4 -in. oil diffusion pump backed by the mechanical pump. Note that the performance of the diffusion pump degrades rapidly at pressures greater than about 0.1 Torr.

This portion of the spectrum contains several low-intensity atomic lines and several band systems. Strong band systems in this region include the $\mathrm{N}_{2}^{+}$first negative system, the $\mathrm{CN}$ violet system and the $\mathrm{CO}$ Herzberg system [34]. Silicon oxide bands from silicone vacuum grease also would appear in this region. When the pressure is reduced from 0.17 to 0.08 Torr using only the mechanical pump, the band intensities increase significantly. A similar pressure dependence for Ar neutral-atom line intensities has been reported using this device [33]. At the same pressure, but with the diffusion pump in use, the emission intensities from the molecular band systems are reduced dramatically without significant changes in the atomic line intensities. The pumping speed of the mechanical pump is quite low at this pressure, and thus with only the mechanical pump in use, the Ar flow was inadequate to purge the chamber of outgassing species and pump oil vapor. The pumping speed of the diffusion pump is much greater at this pressure. For all subsequent studies, the diffusion pump was always used for pressures less than 0.1 Torr.

\subsection{Spatially-resolved emission characteristics}

General features of the low-pressure magnetron glow discharge are illustrated by the spatially-resolved spectra shown in Fig. 3. The top row (a) of wavelength scans shows the Ar neutral-atom lines at 394.90 and $394.75 \mathrm{~nm}$. The middle row (b) shows the Ar ion line at $385.06 \mathrm{~nm}$, and the bottom row (c) shows the $\mathrm{Cu}$ neutral-atom lines at 320.82 and $319.41 \mathrm{~nm}$ as well as the $\mathrm{Cu}$ ion line at $213.60 \mathrm{~nm}$. Table 2 gives information on all emission lines used in this study. The Ar ion-line region was recorded at a ten-fold higher gain setting than the other regions. Note that the $\mathrm{Cu}$ ion line is in the third spectral order while all other lines are in the second order. Spectra are shown from left to right for regions centered 1.0,3.0, 5.0 and $7.0 \mathrm{~mm}$ from the cathode surface.

Both the Ar neutral-atom lines show greatest intensity in the range 3-5 $\mathrm{mm}$ from the cathode surface. The lowest intensity values are observed at a distance of $1 \mathrm{~mm}$ from the cathode. The Ar ion line intensity is greatest for the 3-mm case, and it is smallest for the 7-mm case. Note that the intensities of lines from the support gas 
(a)
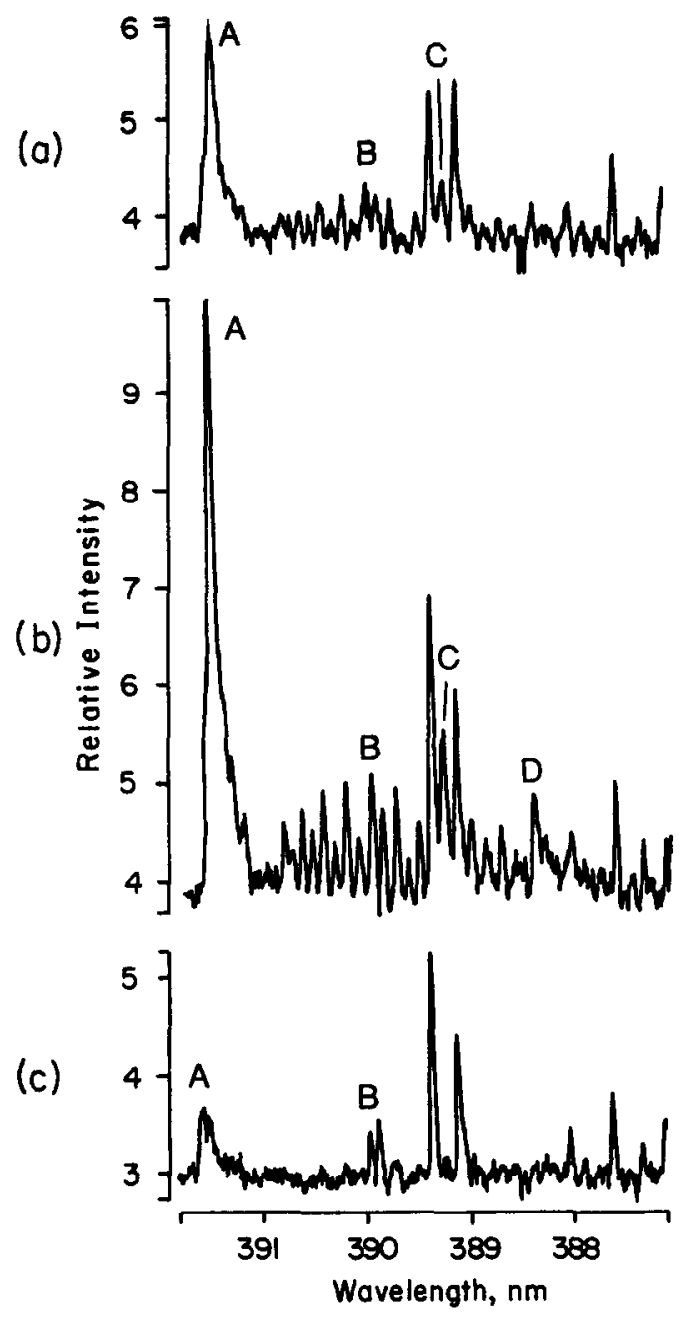

Fig. 2. Magnetron emission spectra in Ar with a $\mathrm{Cu}$ cathode and a $200 \mathrm{~mA}$ discharge current. (a) 0.17 Torr pumped by mechanical pump only; (b) 0.08 Torr pumped by mechanical pump only; (c) 0.08 Torr pumped by 4 -in. diffusion pump backed by mechanical pump. Optical monitoring window centered $3 \mathrm{~mm}$ from cathode surface. A, 391.44-nm head of first negative $\mathrm{N}_{2}^{+}$system; B, $390.21 \mathrm{~nm}$ head of SiO system; C, $389.31 \mathrm{~nm}$ head of Herzberg CO system; D, $388.39 \mathrm{~nm}$ head of violet CN system.

species decrease rather slowly with increasing distance from the cathode surface. This suggests that energetic electrons are found quite far from the cathode surface. For the magnet configuration used here, the peak magnetic field strength at the cathode surface is about $1.1 \mathrm{kG}$. At a distance of $10 \mathrm{~mm}$ from the cathode surface, the peak field strength is about $0.3 \mathrm{kG}$. This is sufficient for plasma operations in the magnetron mode $[35,36]$.

The $\mathrm{Cu}$ neutral-atom line intensities are greatest for the observation zone closest to the cathode surface. Again, the decrease in intensity with increasing distance from the cathode is rather gradual. The $\mathrm{Cu}$ ion line intensity is greatest for the $5-\mathrm{mm}$ case, but the range of intensity values is relatively small for the range of distances from the cathode surface considered here. These data suggest that the plasma is relatively homogeneous over a considerable distance in the direction normal to the cathode surface. There is no evidence of a cathode dark space which is usually observed in glow-discharge plasmas [37]. Except for the electrode surfaces, the plasma does not appear to have a sharp boundary. 
Table 2. Emission line wavelengths and excitation energies

\begin{tabular}{lcc}
\hline Species & $\begin{array}{c}\text { Wavelength } \\
(\mathrm{nm})\end{array}$ & $\begin{array}{c}\text { Excitation potential } \\
(\mathrm{eV})\end{array}$ \\
\hline $\mathrm{Ar}$ & 394.9 & 14.68 \\
& 394.75 & 14.68 \\
& 415.86 & 14.53 \\
$\mathrm{Ar}^{+}$ & 385.06 & 39.70 \\
& 413.17 & 37.07 \\
$\mathrm{Cu}$ & 320.82 & 5.50 \\
& 319.41 & 5.52 \\
& 324.75 & 3.82 \\
$\mathrm{Cu}^{+}$ & 406.27 & 6.87 \\
$\mathrm{Zn}$ & 213.6 & 13.52 \\
$\mathrm{Al}$ & 213.86 & 5.80 \\
$\mathrm{Mn}$ & 396.15 & 3.14 \\
\hline
\end{tabular}

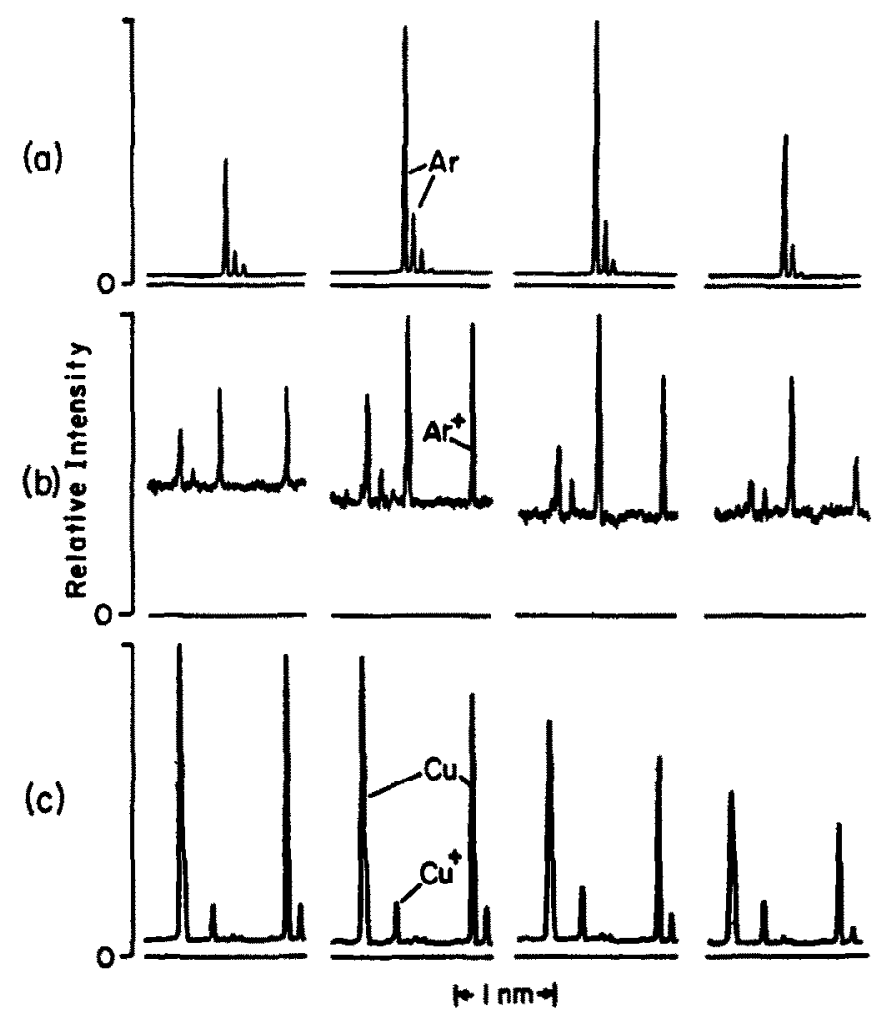

Fig. 3. Spectra from the planar magnetron device using a $\mathrm{Cu}$ cathode. an Ar pressure of 0.01 Torr and a plasma current of $200 \mathrm{~mA}$. Spectra (a), (b) and (c) show spectral regions containing Ar neutral-atom lines, Ar ion lines and $\mathrm{Cu}$ neutral-atom and ion lines, respectively. From left to right, the spectra are from observation zones located $1.0 \mathrm{~mm}, 3.0 \mathrm{~mm}, 5.0 \mathrm{~mm}$ and $7.0 \mathrm{~mm}$ from the cathode surface, respectively.

Figure 4 shows line intensity values (a) and continuum background intensity values (b) plotted vs distance from the cathode surface for the $\mathrm{Cu} 324.7-\mathrm{nm}$ neutral-atom line. The background data were obtained in a line-free region near $324 \mathrm{~nm}$. The plots labeled A, B, C and D correspond to plasma currents of $100,200,300$ and $400 \mathrm{~mA}$, respectively. All data were obtained at a pressure of 0.0007 Torr. This is near the 


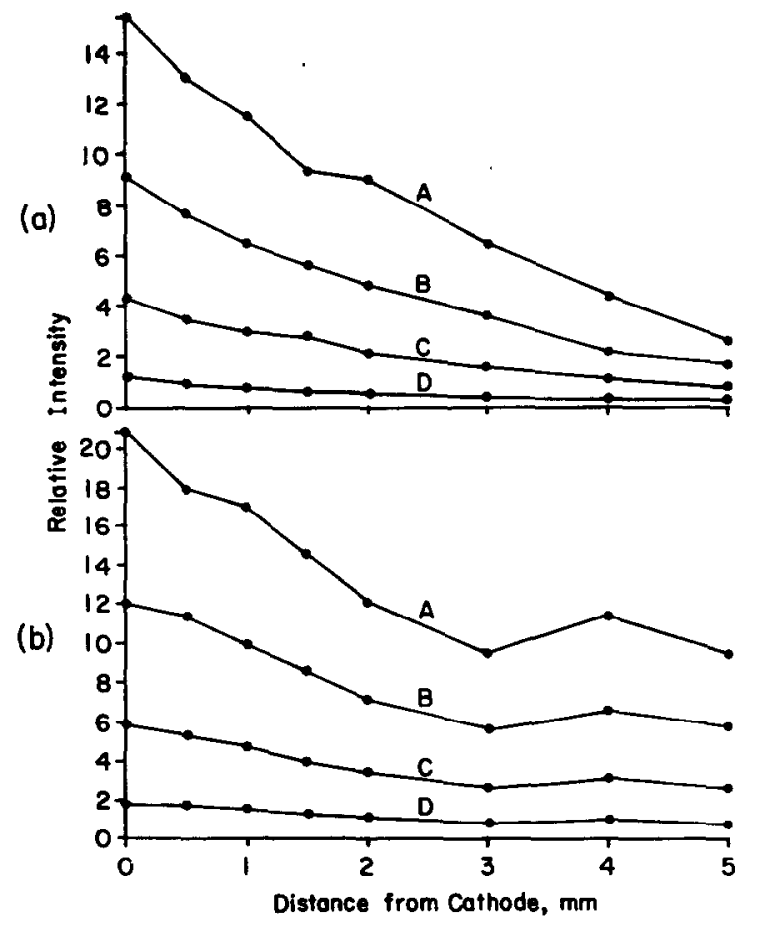

Fig. 4. Intensity vs distance from the cathode surface for the $\mathrm{Cu} 324.7-\mathrm{nm}$ neutral-atom line (a) and for continuum background (b) at an Ar pressure of 0.0007 Torr. A, $400 \mathrm{~mA} ; \mathrm{B}, 300$ $\mathrm{mA}$; C, $200 \mathrm{~mA}$; D, $100 \mathrm{~mA}$.

lower end of the usual pressure range for the device described here. Similar data for pressures of 0.01 and 2.5 Torr are shown in Figs 5 and 6, respectively. Note that 0.01 Torr is typical of the pressures used with planar magnetron systems designed for vapordeposited film manufacturing [27,28]; while 2.5 Torr is more typical of the pressures used with glow discharge devices designed for analytical atomic spectroscopy $[6,7]$.

For all pressure and current values investigated, both $\mathrm{Cu}$ line radiation and continuum background radiation are detected very near the cathode surface. Again, this suggests the absence of a well-defined cathode dark space. In a conventional glow discharge, electrons liberated from the cathode surface by positive ion bombardment are accelerated away from the cathode and may travel a considerable distance before undergoing inelastic collisions with cathodically sputtered vapor and plasma gas species. This distance increases with decreasing pressure, and thus the width of the cathode dark space is very pressure dependent $[37,38]$.

In the planar magnetron configuration, the electron trajectories are bent by the magnetic field, and in the absence of collisions, the trajectories must follow the curved magnetic field lines. Since these field lines pass through the cathode surface at both ends [28], the electrons may spend a significant amount of time in the region near the cathode surface. Note that for both the 0.0007 Torr case (Fig. 4) and the 0.01 Torr case (Fig. 5) the greatest $\mathrm{Cu}$ line and background intensity values occur in the measurement zone closest to the cathode surface. However, for the 2.5 Torr case intensity values for the lower currents are greatest between 1 and $2 \mathrm{~mm}$ from the cathode surface.

At the lowest pressure, the electron mean free path is of the order of $50 \mathrm{~m}$, and it is unlikely that any radiative processes involving electron impact would be observed if it were not for the fact that the electrons are trapped by the presence of the magnetic field. At higher pressures, collisional scattering reduces electron trapping efficiency, and increased electron diffusion and mobility may result in an expansion of the plasma in the direction away from the cathode surface, possibly explaining the greater intensity values observed 1 and $2 \mathrm{~mm}$ from the cathode surface. 


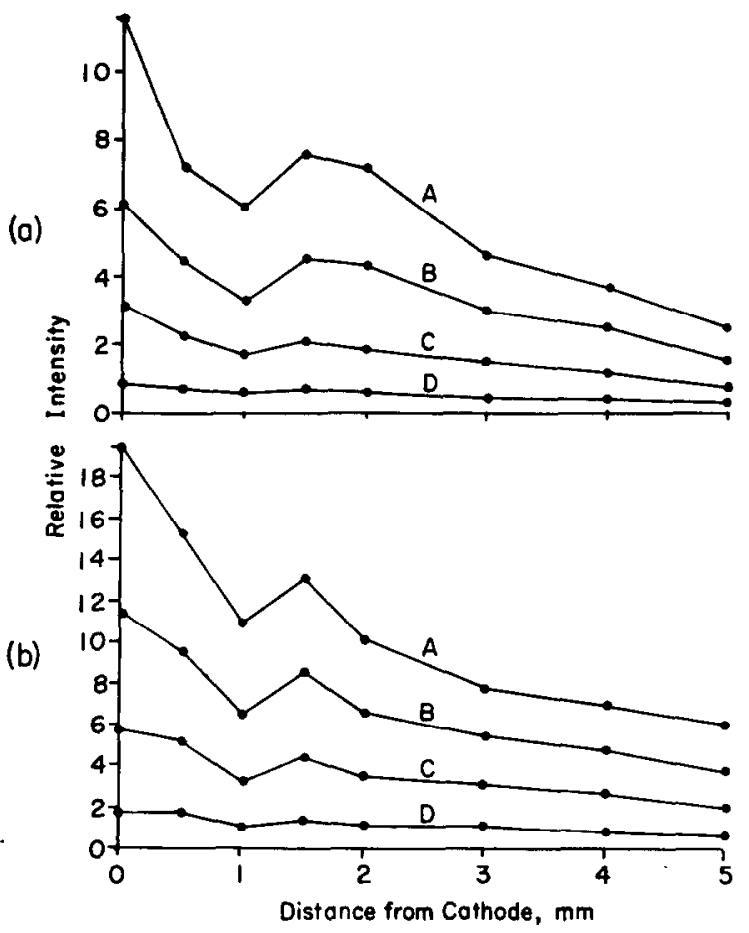

Fig. 5. Intensity vs distance from the cathode surface for the $\mathrm{Cu} 324.7-\mathrm{nm}$ neutral-atom line (a); and for continuum background (b) at an Ar pressure of 0.01 Torr. A. $400 \mathrm{~mA}$; B. 300 $\mathrm{mA} ; \mathrm{C}, 200 \mathrm{~mA}$; D, $100 \mathrm{~mA}$.

At all three pressures, the continuum background intensity decreases relatively slowly with increasing distance from the cathode surface. Some relatively weak structural features are observed in the continuum background plots for all three pressures. For the 0.0007 -Torr case, a secondary zone of slightly increased intensity is observed at a distance for about $4 \mathrm{~mm}$ for all four current values. For the 0.01 -Torr case, this zone occurs closer to the cathode surface. For the 2.5-Torr case, this zone is less apparent.

The intensity scales for Figs 4-6 are comparable. Note that the $\mathrm{Cu}$ line intensity values are largest at a pressure of 0.0007 Torr and smallest at a pressure of 2.5 Torr. Values typically are about a factor-of-three greater at the lower pressure. Continuum background intensity shows a weaker dependence on gas pressure than the $\mathrm{Cu}$ line intensity. For most current-pressure combinations, the highest background values are observed for the 0.0007-Torr case.

At a pressure of 0.0007 Torr, the $\mathrm{Cu}$ neutral-atom line intensity plots show relatively little structure, and intensity values decrease gradually with increasing distance from the cathode surface. At 0.01 Torr, intensity values decrease more sharply in the first millimeter from the cathode surface, and a well-defined hump is observed in the plots in the region from $1-3 \mathrm{~mm}$ from the cathode surface. This is observed for all current values. At 2.5 Torr, the $\mathrm{Cu}$ line intensity vs distance plots show two distinct regions. In the region from $0-2 \mathrm{~mm}$ from the cathode surface, the shape of the plots varies with plasma current, and for the lower current values, peak intensity values are observed at about $1 \mathrm{~mm}$ from the cathode. This is the only case where there is any indication of a cathode dark space, suggesting that the device may not be operating in the magnetron mode [32,33]. For distances greater than about $2 \mathrm{~mm}$ from the cathode surface, the 2.5-Torr plots are similar to those from the other pressures.

Line-to-background intensity ratios at 0.0007 Torr show relatively little variation with distance from the cathode for distances less than about $3 \mathrm{~mm}$. For greater distances, the ratios decrease sharply with increasing distance. Line-to-background ratios are nearly independent of plasma current for currents in the range $200-400 \mathrm{~mA}$ 


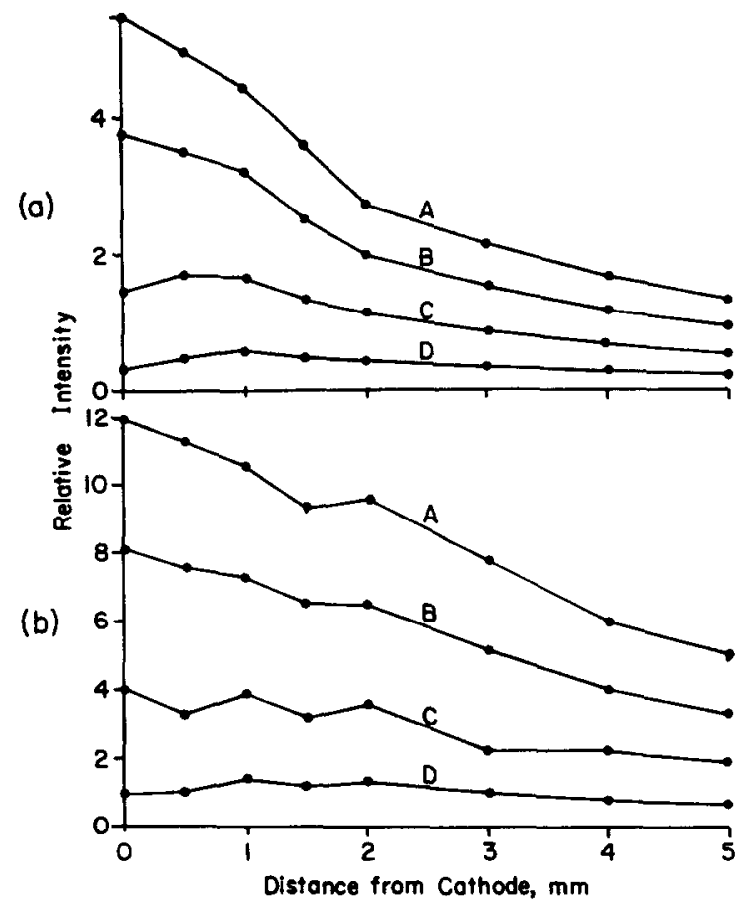

Fig. 6. Intensity vs distance from the cathode surface for the $\mathrm{Cu} 324.7-\mathrm{nm}$ neutral-atom line (a); and for continuum background (b) at an Ar pressure of 2.5 Torr. A, $400 \mathrm{~mA}$; B, 300 $\mathrm{mA}$; C, $200 \mathrm{~mA}$; D, $100 \mathrm{~mA}$.

at all distances from the cathode surface considered in this study. Significantly lower line-to-background ratios are obtained at $100 \mathrm{~mA}$.

At 0.01 Torr, peak line-to-background ratios are observed about $2 \mathrm{~mm}$ from the cathode surface for the 300 and $400 \mathrm{~mA}$ cases. At this pressure, line-to-background ratios increase steadily with increasing plasma current for the range of values used here. At 2.5 Torr, the largest line-to-background ratios occur about $1 \mathrm{~mm}$ from the cathode surface, and values decrease rapidly with increasing distance in the range 1-2 $\mathrm{mm}$ from the surface. Line-to-background ratios are relatively independent of current for the range of values used here.

In general, the $\mathrm{Cu}$ line-to-background ratios are greatest in the range 0-2 $\mathrm{mm}$ from the cathode. Within this range, values do not vary with distance by more than a factor of two for any of the pressures investigated. In addition, the effects of plasma current on line-to-background ratios are relatively small for values in the range $100-400 \mathrm{~mA}$.

\subsection{Pressure and current sensitivity of emission intensities}

Figure 7 shows the effects of Ar pressure on the emission intensities from an Ar neutral-atom line (A), continuum background (B), a Cu neutral-atom line (C), an Ar ion line (D) and a $\mathrm{Cu}$ ion line (E). In all cases, the plasma current was $400 \mathrm{~mA}$. All measurements were made in the region centered about $0.35 \mathrm{~mm}$ from the cathode surface. Note that these data represent more than a 3500-fold pressure range.

The Ar neutral-atom intensity vs pressure plot is unique relative to the other plots in that the intensity shows a monotonic increase with decreasing pressure over almost the entire pressure range. This increase is small in the pressure range $0.1-2.5$ Torr and in the range $0.0007-0.005$ Torr. At intermediate pressures in the range $0.01-0.1$ Torr, the Ar neutral-atom line intensity shows a larger pressure sensitivity.

The continuum background intensity is greatest at a pressure of about 1.0 Torr. As the pressure is reduced, the background first decreases rapidly and then becomes nearly pressure independent in the range $0.005-0.05$ Torr. With further decreases in pressure, the background intensity increases slowly at first and then more rapidly as 


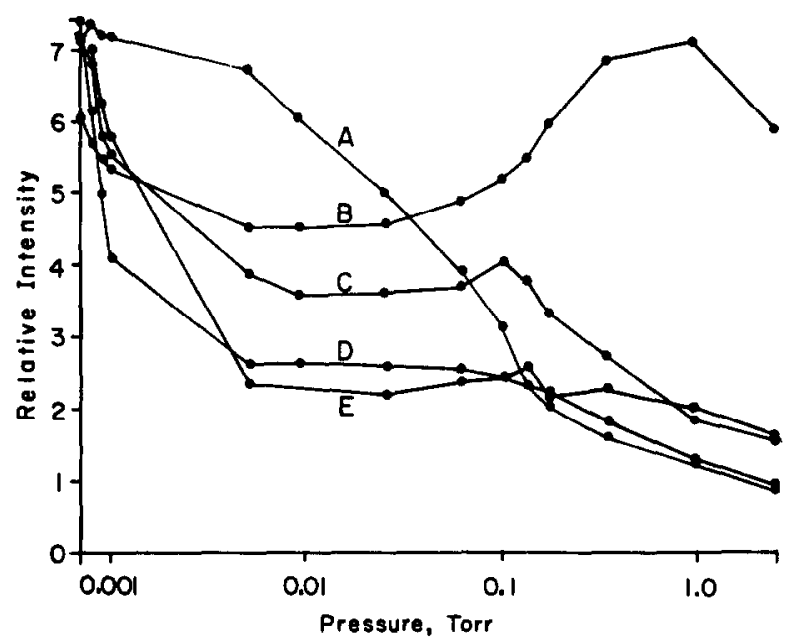

Fig. 7. Intensity vs pressure plots using a $\mathrm{Cu}$ cathode and a $400 \mathrm{~mA}$ plasma current. $\mathrm{A}, \mathrm{Ar}$ 415.86-nm neutral-atom line; B, continuum background near $406 \mathrm{~nm} ; \mathrm{C}, \mathrm{Cu} 406.27-\mathrm{nm}$ neutralatom line; $\mathrm{D}, \mathrm{Ar} 413.17-\mathrm{nm}$ ion line; $\mathrm{E}, \mathrm{Cu} 213.6-\mathrm{nm}$ ton line. All data were obtained from an observation window centered $0.35 \mathrm{~mm}$ from the cathode surface.

the low-pressure operation limit is approached. The range of background intensity values is really quite small considering the very large pressure range.

The $\mathrm{Cu}$ neutral-atom line plot as well as the plots for the ion lines of $\mathrm{Ar}$ and $\mathrm{Cu}$ shows three more-or-less distinct pressure regions. As the pressure is reduced from 2.5 Torr, intensities from these species first increase until a pressure of about 0.1 Torr is reached. This increase is larger for the $\mathrm{Cu}$ neutral-atom and $\mathrm{Ar}$ ion lines. In the pressure range $0.005-0.1$ Torr, the intensity values are remarkably independent of gas pressure. For pressures less than about 0.005 Torr, the intensity values increase with decreasing pressure. This increase is quite dramatic for pressures less than 0.001 Torr and is largest for the $\mathrm{Ar}$ and $\mathrm{Cu}$ ion lines. Note that in all cases, intensity values are greatest at the lowest attainable pressure.

The data in Fig. 7 suggest that the magnetron glow discharge plasma has several stable operating modes. For the higher pressures used here, electron mean free paths are much less than the mean circumference of the plasma ring. Thus, electron path length amplification is not significant. However, the magnetic field does control the plasma size and shape. In addition, the presence of the magnetic field reduces the rate of electron diffusion in the direction normal to the magnetic field. In turn, this may reduce the plasma voltage.

The data suggest that a transition to a different mode occurs in the pressure range 0.05-0.2 Torr. Note that this is the pressure range showing the greatest pressure sensitivity of both the Ar neutral-atom line intensity and the continuum background intensity. Also note that the $\mathrm{Cu}$ neutral-atom and ion line plots show discontinuities in this pressure range. Previously reported voltage vs pressure plots for this system showed kinks in the pressure range $0.1-0.2$ Torr $[32,33]$.

For pressures less than about 0.05 Torr, the mean free path of a $100 \mathrm{eV}$ electron is comparable to the plasma-ring diameter. From about this pressure to 0.005 Torr, the plasma may be operating in the magnetron mode with significant electron path length amplification. In this region, only the Ar neutral-atom line intensities show a significant pressure sensitivity. This is the pressure range usually used with magnetron devices designed as high-efficiency vapor sources for thin film manufacturing [27,28].

At pressures less than about 0.005 Torr, all the plots except neutral-atom Ar show dramatic intensity increases with decreasing pressure. The Ar neutral-atom line intensity values, however, become nearly pressure independent at the lowest pressures. At the lowest pressures, the low availability of Ar atoms may be responsible for the patterns observed in Fig. 7. 


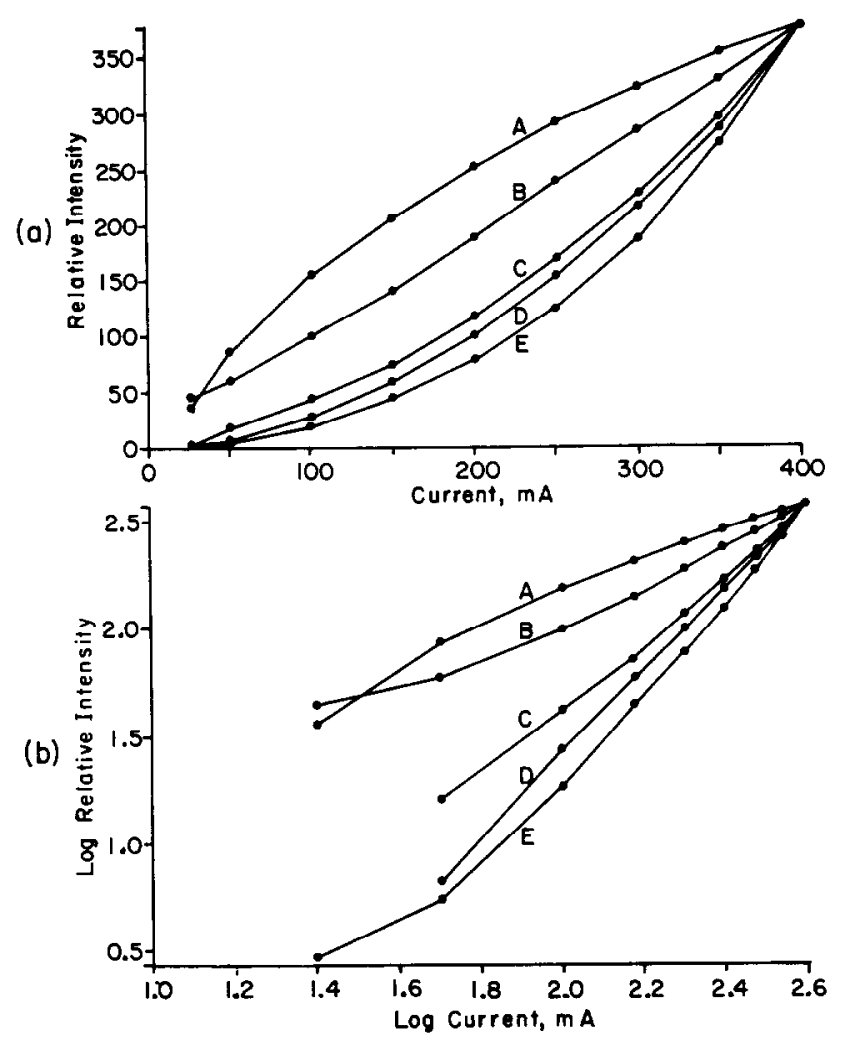

Fig. 8. Intensity vs current plots on a linear scale (a) and on a $\log -\log$ scale (b) using a $\mathrm{Cu}$ cathode and an Ar pressure of 0.001 Torr. A, Ar 415.86-nm neutral-atom line; $\mathrm{B}, \mathrm{Ar} 413.17$ $\mathrm{nm}$ jon line; $C$, continuum background near $406 \mathrm{~nm} ; \mathrm{D}, \mathrm{Cu} 406.27-\mathrm{nm}$ neutral-atom line; $\mathrm{E}$, $\mathrm{Cu}$ 213.6-nm ion line. All data were obtained from an observation window centered $0.35 \mathrm{~mm}$ from the cathode surface.

Figure 8 shows the effects of plasma current on the emission intensities from an $\mathrm{Ar}$ neutral-atom line (A), and an $\mathrm{Ar}$ ion line (B), continuum background (C), a $\mathrm{Cu}$ neutral-atom line (D) and a $\mathrm{Cu}$ ion line (E). The upper set of plots (a) is presented on a linear scale; while the lower set (b) is presented on a log-log scale. In all cases, the Ar pressure was 0.001 Torr. All measurements were made in the region centered about $0.35 \mathrm{~mm}$ from the cathode surface. Because of the large intensity range for the different lines monitored here, the intensity values for each plot were normalized to give the same value at the highest current value used. This greatly facilitates comparison of the current sensitivities for the different species.

The plot for the Ar neutral-atom line is sub-linear over the entire current range. The $\mathrm{Ar}$ ion line plot is more nearly linear. The $\mathrm{Cu}$ ion-line intensity shows the greatest sensitivity to the discharge current. Since both the $\mathrm{Cu}$ neutral-atom and ion lines show a greater current sensitivity than the continuum background, higher current values should result in greater powers of detection for these emission lines.

ROSSNAGEL and SAENGER [39] have developed a model for the current sensitivity of emission line intensities in a planar magnetron glow discharge. The model is based on the validity of the Langmuir-Child law [40] in the space-charge sheath near the cathode surface. The model predicts that local electron densities in the plasma are proportional to the discharge current. Then, emission processes requiring one electron collision, such as excitation of $\mathrm{Ar}$ atoms, should show a linear current sensitivity. Emission processes requiring two electron collisions should have intensity values proportional to the square of the discharge current. This includes the sequential impact ionization and excitation of Ar atoms and the impact excitation of atoms sputtered by $\mathrm{Ar}$ ion bombardment. 
The usefulness of this model can be ascertained from the slopes of the log-log plots in Fig. 8(b). Note that the log-log plots all become more linear at the higher current values. For the $\mathrm{Cu}$ neutral-atom line emission (plot $\mathrm{D}$ ), the slope is 1.93 . While this is in good agreement with the model, the $\log -\log$ slopes for the other emission lines are significantly lower than the values predicted by the model. For the Ar neutralatom line, gas rarefaction effects from the heat generated near the cathode surface may result in a much lower Ar density near the cathode at the higher current values [41]. This would decrease the current sensitivity. The log-log slope for the Ar ion line at the higher current values is about 1.0; while the predicted value is 2.0 . The lower observed value may be the result of gas rarefaction effects as well as the direct formation of electronically-excited ions by single collisions with fast electrons. The $\mathrm{Cu}$ ion line slope is about 2.3; the predicted value is 3.0. Again, the formation of electronically-excited ions from single collisons would reduce the current sensitivity from the predicted value.

Figure 9 shows plots of intensity vs pressure from a $\mathrm{Zn}$ alloy pellet (NIST SRM 630) positioned to intersect the sputter track (see Fig. 1). The upper set of plots (a) was obtained for the $\mathrm{Zn}$ 213.86-nm neutral-atom line, and the lower set (b) was obtained for the A1 396.15-nm neutral-atom line. The $\mathrm{Al}$ is present in the $\mathrm{Zn}$ at the $4.30 \%$ level. For both sets, plots A, B, C and D were obtained with current values of $400,300,200$ and $100 \mathrm{~mA}$, respectively. All measurements were made using an observation window located $0.35 \mathrm{~mm}$ from the cathode surface.

The $\mathrm{Zn}$ neutral-atom line plots have some features similar to the $\mathrm{Cu}$ neutral-atom line plots in Fig. 7. Note that a discontinuity in the plots is observed at a pressurc of about 0.1 Torr. For pressures in the range $0.005-0.05$ Torr, the $\mathrm{Zn}$ intensity shows relatively little pressure sensitivity. For pressures less than about 0.005 Torr, the $\mathrm{Zn}$ intensity increases with decreasing pressure, but the increase is not as dramatic as for the $\mathrm{Cu}$ line in Fig. 7.

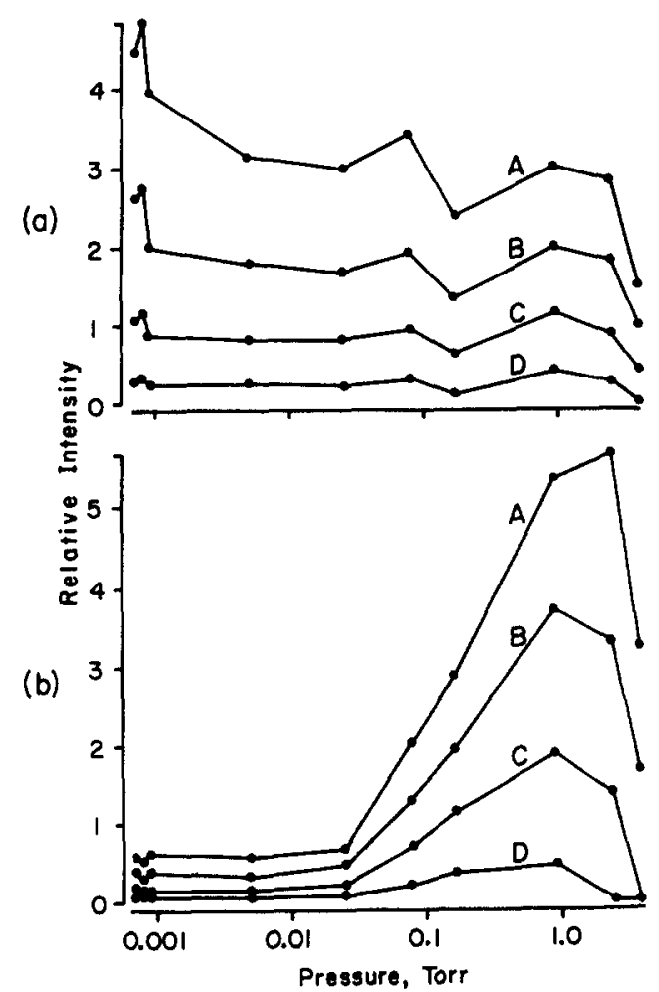

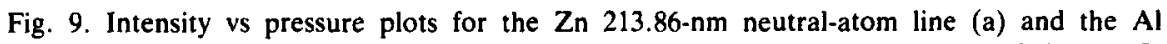
396.15-nm neutral-atom line (b) from a $\mathrm{Zn}$-alloy sample pellet. A, $400 \mathrm{~mA} ; \mathrm{B}, 300 \mathrm{~mA}$; C, $200 \mathrm{~mA} ; \mathrm{D}, 100 \mathrm{~mA}$. All data were obtained from an observation window centered $0.35 \mathrm{~mm}$ from the cathode surface. 
The pressure sensitivity of the Al neutral-atom line is very different, showing a very pronounced local maximum at a pressure of about 1 Torr. The intensity decreases gradually with decreasing pressure down to about 0.05 Torr, and then becomes nearly pressure-independent at still lower pressures. The intensity decrease from 1 Torr to 0.01 Torr is about an order of magnitude. Preliminary studies with $\mathrm{Mn}$ in the same $\mathrm{Zn}$ alloy pellet show behavior which is qualitatively similar to that observed for the Al. A more detailed characterization of these $\mathrm{Zn}$-alloy standard reference materials with the planar magnetron glow discharge is in progress. Overall, there appears to be little correlation between line intensities, ionization potentials and excitation potentials, probably because thermal processes do not predominate in the plasmas described in this report [42].

\section{Conclusions}

The planar magnetron glow discharge is a unique device with potentially attractive features for chemical analysis. The device operates over an extraordinary range of pressures and current densities with excellent stability. The use of very low pressures is attractive for several reasons. First, spectral interferences, particularly bands from residual gas species, can be significantly reduced when adequate pumping speeds are used. This should be useful for atomic mass spectrometry as well as for emission spectroscopy applications. For the latter applications, the highest line-to-background ratios are observed at low pressures for neutral-atom lines of $\mathrm{Fe}, \mathrm{Mg}$ and $\mathrm{Zn}$. Second, greatly reduced back-scattering of sputtered material should increase both the speed and reliability of depth profiling techniques using positive ion sputtering. Third, interfacing the low-pressure plasma to a mass spectrometer may be simpler and may permit the use of smaller pumps. Evaluation of such a system is currently underway in this laboratory. Finally, the planar magnetron provides a means for the study of basic sampling, excitation and ionization processes under a very wide range of neutralgas pressures.

The structure and properties of the plasma also are unique. The structure of the plasma shows only relatively small changes over a 3500 -fold range of pressures. The size, shape and location of the plasma appear to be controlled primarily by the shape and amplitude of the magnetic field with neutral-gas pressure and current density being much smaller factors. The plasma appears relatively homogeneous with respect to excitation and ionization conditions with relatively energetic species observed at all distances from the cathode investigated in this study. Over most of the operating pressure range, no cathode dark space is observed. Nearly comparable values of analysis line-to-background ratios are observed for regions from the cathode surface to at least $2 \mathrm{~mm}$ from the surface. This makes optical alignment and positioning of the sample quite straightforward.

The pressure and current sensitivities of line emission intensities are quite complex, and significantly more study is needed. In general, a reduction in neutral-gas pressure or an increase in current density results in increased emission from species with greater internal energy. Only the $\mathrm{Al}$ and $\mathrm{Mn}$ neutral-atom lines, with excitation energies of 3.14 and $3.08 \mathrm{eV}$, respectively, showed lower intensities at the lower pressures. The ion lines investigated here showed very large current and pressure sensitivities, and the use of pressures less than 0.001 Torr with high current densities may be useful for efficient ion production. A planar magnetron, similar to the one described here, recently has been interfaced to a quadrupole mass spectrometer, and spatially-resolved ion density measurements will be used to provide more direct information on the size, shape and structure of the plasma as well as to determine its usefulness as an ion source for analytical mass spectrometry. 


\section{REFERENCES}

[1] C. L. Chakrabarti, K. L. Hendrick, J. C. Hutton, Z. Bicheng, P. C. Bertels and M. H. Beck, Anal. Chem. 62, 547 (1990).

[2] D. S. Gough, Anal. Chem. 48, 1926 (1976).

[3] B. J. Russell and A. Walsh, Spectrochim. Acta 15B, 883 (1959).

[4] B. W. Smith, N. Omenetto and J. D. Winefordner, Spectrochim. Acta 39B, 1389 (1986).

[5] D. S. Gough, P. Hannaford and A. Walsh, Spectrochim. Acta 28B, 197 (1973).

[6] K. Wagatsuma and K. Hirokawa, Anal. Chem. 60, 702 (1988).

[7] M. E. Waitlevertch and J. K. Hurwitz, Appl. Spectrosc. 30, 510 (1976).

[8] C. J. Belle and J. D. Johnson, Appl. Spectrosc. 27, 118 (1973).

[9] S. El Alfy, K. Laqua and H. Massmann, Fresenius' Z. Anal. Chem. 263, 1 (1973).

[10] W. Grimm, Spectrochim. Acta 23B, 443 (1968).

[11] W. W. Harrison and B. L. Bentz, Prog. Anal. Spectrosc. 11, 53 (1988).

[12] W. W. Harrison, K. R. Hess, R. K. Marcus and F. L. King, Anal. Chem. 58, 341A (1986).

[13] D. C. Duckworth and R. K. Marcus, Appl. Spectrosc. 44, 649 (1990).

[14] K. R. Hess and W. W. Harrison, Anal. Chem. 58, 1696 (1986).

[15] J. W. Coburn and W. W. Harrison, Appl. Spectrosc. Rev. 17, 95 (1981).

[16] P. R. Banks and M. W. Blades, Spectrochim Acta 44B, 1117 (1989).

[17] M. R. Winchester and R. K. Marcus, Appl. Spectrosc. 42, 941 (1988).

[18] H. J. Kim and E. H. Piepmeier, Anal. Chem. 60, 2040 (1988).

[19] D. C. Duckworth and R. K. Marcus, Anal. Chem. 61, 1897 (1989).

[20] M. Glick, B. W. Smith and J. D. Winfordner, Anal. Chem. 62, 157 (1990).

[21] S. Tanguay and R. Sacks, Appl. Spectrosc. 42, 576 (1988).

[22] R. A. Kruger, R. M. Bombelka and K. Laqua, Spectrochim. Acta 35B, 581 (1980).

[23] B. L. Bentz and W. W. Harrison, Anal. Chem. 54, 1644 (1982).

[24] R. N. Franklin, Plasma Phenomena in Gas Discharges. Clarendon Press, Oxford (1976).

[25] R. Simonneau and R. Sacks, Appl. Spectrosc. 43, 141 (1989).

[26] F. F. Chen, Introduction to Plasma Physics and Controlled Fusion, 2nd Edn, Vol. 1. Plenum Press, New York (1984).

[27] J. A. Thornton and A. S. Penfold, Cylindrical magnetron sputtering, in Thin Film Processes, Eds, J. L. Vossen and W. Kern. Academic Press, New York (1978).

[28] R. K. Waits, Planar magnetron sputtering, in Thin Film Processes, Eds, J. L. Vossen and W. Kern. Academic Press, New York (1978).

[29] A. R. Nyaiech, Thin Solid Films 86, 267 (1981).

[30] S. Schiller, V. Heisig and K. Goedicke, J. Vac. Sci. Technol. 14, 815 (1977).

[31] S. Schiller, V. Heisig and K. Goedicke, Thin Solid Films 54, 33 (1978).

[32] L. McCaig, R. Sacks and D. Lubman, Appl. Spectrosc. 43, 912 (1989).

[33] L. McCaig, N. Sesi and R. Sacks, Appl. Spectrosc. 44, 1176 (1990).

[34] R. W. B. Pearse and A. G. Gaydon, The Identification of Molecular Spectra, 4th Edn. Chapman and Hall, New York (1976).

[35] F. A. Green and B. N. Chapman, J. Vac. Sci. Technol. 13, 165 (1976).

[36] A. G. Spencer, C. A. Bishop and R. P. Howsen, Vacuum 37, 363 (1981).

[37] M. E. Pillow, Spectrochim. Acta 36B, 821 (1981).

[38] A. von Engel, lonized Gases. Clarendon Press, Oxford (1965).

[39] S. M. Rossnagel and K. L. Saenger, J. Vac. Sci. Technol. A7, 968 (1989).

[40] C. D. Child, Phys Rev. 32, 492 (1911).

[41] S. M. Rossnagel, J. Vac. Sci. Technol. A6, 19 (1988).

[42] H. Falk, Ann. Phys. 7.F. 16, 160 (1965). 